\title{
Linearizing bad sequences: upper bounds for the product and majoring well quasi-orders
}

\author{
Sergio Abriola ${ }^{1}$, Santiago Figueira ${ }^{2,3 \star}$, and Gabriel Senno ${ }^{2}$ \\ 1 Dto. Matemática, FCEN, Universidad de Buenos Aires, Argentina \\ 2 Dto. Computación, FCEN, Universidad de Buenos Aires, Argentina \\ 3 CONICET, Argentina
}

\begin{abstract}
Well quasi-orders (wqo's) are an important mathematical tool for proving termination of many algorithms. Under some assumptions upper bounds for the computational complexity of such algorithms can be extracted by analyzing the length of controlled bad sequences. We develop a new, self-contained study of the length of bad sequences over the product ordering of $\mathbb{N}^{n}$, which leads to known results but with a much simpler argument.

We also give a new tight upper bound for the length of the longest controlled descending sequence of multisets of $\mathbb{N}^{n}$, and use it to give an upper bound for the length of controlled bad sequences in the majoring ordering of sets of tuples. We apply this upper bound to obtain complexity upper bounds for decision procedures of automata over data trees.

In both cases the idea is to linearize bad sequences, i.e. transform them into a descending one over a well-order for which upper bounds can be more easily handled.
\end{abstract}

\section{Introduction}

A quasi-order is a binary relation $\leq$ over a given set $A$ that is reflexive and transitive. A sequence $\mathbf{X}=x_{0}, x_{1}, x_{2}, \ldots$ of elements of $A$ is called good if there are $i<j$ such that $x_{i} \leq x_{j}$. A sequence is bad if it is not good. A well quasi-order (wqo) is a quasi-order where all infinite sequences are good, or, equivalently, all bad sequences are finite.

Wqo's are widely used in termination proofs of algorithms in constraint solving, automated deduction, program analysis, verification and model checking, logic, etc. From the analysis of a termination proof of a given algorithm $\mathcal{S}$, whose correctness is grounded in the analysis of certain wqo, one may extract a computational complexity upper bound for $\mathcal{S}$. Roughly, the idea is that any sequence of successive configurations of $\mathcal{S}$ (with a given input) is transformed into a bad sequence in the wqo. Thus, having an upper bound for the length of the bad sequence entails an upper bound for the number of steps that the algorithm needs to terminate.

\footnotetext{
* Figueira was partially supported by UBA (UBACyT 20020090200116), ANPCyT (PICT-2010-0688) and CONICET (PIP 370).
} 
However, in principle, a bad sequence over a wqo can be arbitrarily large. For instance, the lexicographic ordering $\leq_{\text {lex }}$ over $\mathbb{N}^{n}$ is a well-order, and hence a wqo. Observe that for $\mathbb{N}^{2}$ and any $N$, the sequence

$$
\langle 1,0\rangle,\langle 0, N\rangle,\langle 0, N-1\rangle,\langle 0, N-2\rangle, \ldots,\langle 0,1\rangle,\langle 0,0\rangle
$$

is $\leq_{\text {lex }}$-bad (which in a total order is equivalent to say that it is decreasing) and has length greater than $N$. Therefore, in general there is no bound to the length of a bad sequence starting with a given element: bad sequences in a wqo are finite but could be arbitrarily large.

In practice, in the analysis of termination proofs, one has two additional assumptions of a wqo $(A, \leq)$. First, one has some effective way of measuring the size of each element $x \in A$, notated $|x|_{A}$ or simply $|x|$.

Definition 1. [13] $A$ norm function $|\cdot|_{A}$ over a set $A$ is a mapping $|\cdot|_{A}: A \rightarrow \mathbb{N}$ that provides every element of $A$ with a positive integer, its norm. The norm function is said to be proper if $\left\{\left.x \in A|| x\right|_{A}<n\right\}$ is finite for every $n$.

Second, we may restrict ourselves to bad sequences $\mathbf{x}=x_{0}, x_{1}, x_{2} \ldots$ with a controlled behavior, which means that there is an effective way of computing, given $i$, an upper bound for $\left|x_{i}\right|$.

Definition 2. Let $g: \mathbb{N} \rightarrow \mathbb{N}$ be a computable increasing function and let $(A, \leq)$ be a wqo with a proper norm. A bad sequence $\mathbf{x}=x_{0}, x_{1}, x_{2} \ldots$ is $g$, $t$-controlled if for all $i,\left|x_{i}\right|_{A}<g(t+i)$. We say that $g$ is the control function for $\mathbf{x}$.

As a consequence of König's Lemma, controlled bad sequences over wqos cannot be arbitrarily large: given a control, there exist upper bounds for their lengths. Let us go back to the example of the $\leq_{\text {lex }}$-decreasing sequence in (1). If we further impose that the sequence is $g, 0$-controlled, where $g(0)=2$ and we fix $|x|_{\mathbb{N}^{2}}$ to be the infinity norm of $x$ then the reader may verify that the longest $g, 0$-controlled decreasing sequence is

$$
\langle 1,1\rangle,\langle 1,0\rangle,\langle 0, g(2)-1\rangle,\langle 0, g(2)-2\rangle, \ldots,\langle 0,1\rangle,\langle 0,0\rangle .
$$

In this paper we give upper bounds for the length of $g, t$-controlled bad sequences, when $t$ is a parameter. That is, given a well (quasi) order under study (we address lexicographic, product, multiset and majoring) $(A, \leq)$, we define $L_{g}^{A}(t)$ as the length of the longest $g, t$-controlled bad sequence in $(A, \leq)$, and we study upper bounds for $L_{g}^{A}$, which are classified in the Fast Growing Hierarchy $\left(\mathfrak{F}_{\alpha}\right)_{\alpha<\epsilon_{0}}$ of Löb and Wainer [10].

For a more detailed introduction to some topics of this paper, see [1].

\section{Linearizing}

Our technique to obtain an upper bound for $L_{g}^{A}$ is to linearize the wqo $\left(A, \leq_{A}\right)$ with a proper norm $|\cdot|_{A}$ into a suitable well linear order $\left(B, \leq_{B}\right)$ with a proper norm $|\cdot|_{B}$. This means to find a function $h: A^{+} \rightarrow B$ such that for every 
$\mathbf{a} \in A^{+}$and $a \in A$, if $\mathbf{a}^{\Upsilon} a$ is a bad sequence in $\left(A, \leq_{A}\right)$ then $h(\mathbf{a})>_{B} h\left(\mathbf{a}^{\frown} a\right)$. So if $\mathbf{a}=a_{0}, \ldots, a_{k}$ is bad in $\left(A, \leq_{A}\right)$ then

$$
\mathbf{b}=h\left(a_{0}\right), h\left(a_{0}, a_{1}\right), h\left(a_{0}, a_{1}, a_{2}\right), \ldots, h(\mathbf{a})
$$

is descending in $\left(B, \leq_{B}\right)$. Furthermore, for any control function $g$ we seek a control function $\tilde{g}$ such that if $\mathbf{a}$ is $g, t$-controlled then $|h(\mathbf{a})|_{B}<\tilde{g}(|\mathbf{a}|+t-1)$ - here $|\mathbf{a}|$ denotes the length of $\mathbf{a}$. Hence if $\mathbf{a}$ is $g, t$-controlled then $\mathbf{b}$ is $\tilde{g}, t$ controlled and therefore from a $g, t$-controlled bad sequence in $\left(A, \leq_{A}\right)$ one can get a $\tilde{g}, t$-descending sequence in $\left(B, \leq_{B}\right)$ of the same length. Hence $L_{g}^{A} \leq L_{\tilde{g}}^{B}$, and the task is now to find an upper bound for $L_{\tilde{g}}^{B}$. In practice, these upper bounds are easier to devise for well-orders than for wqo's.

\section{Our contributions}

Product and lexicographic ordering. For some dimension $n$, let $\left(\mathbb{N}^{n}, \leq_{\mathrm{pr}}\right)$ be the set of $n$-tuples of $\mathbb{N}$ ordered with the natural product ordering. Dickson's Lemma is the statement that $\left(\mathbb{N}^{n}, \leq_{\mathrm{pr}}\right)$ is a wqo. We denote $L_{n, g}^{\mathrm{pr}}(t)$ the length of the longest $g, t$-controlled bad sequence over $\left(\mathbb{N}^{n}, \leq_{\text {pr }}\right)$. Here we take $|x|_{\mathbb{N}^{n}}$ to be $|x|_{\infty}$.

McAloon [11] shows an upper bound for $L_{n, g}^{\mathrm{pr}}$ when $g$ is linear, and places it at the level $\mathfrak{F}_{n+1}$ of the Fast Growing Hierarchy. Later Clote [2] simplifies McAloon's argument and finds an upper bound in $\mathfrak{F}_{n+6}$. Neither of these proofs are self contained and both are quite complex. In 5 . D. and S. Figueira, Schmitz and Schnoebelen show an improved upper bound of $\mathfrak{F}_{n}$ with a simpler proof, relying in a mathematical more general setting of disjoint unions of powers of $\mathbb{N}$. In fact, the main result is both more general and more precise than those of McAloon and Clote: if $g \in \mathfrak{F}_{\gamma}$ then $L_{n, g}^{\mathrm{pr}}$ is bounded by a function in $\mathfrak{F}_{\gamma+n-1}$. Although this proof is markedly simpler than those of [11] and [2], there are still some technical lemmas regarding this richer setting.

In Thm. 4 we give an even shorter, elementary and self-contained proof of the result of [5] which only uses a linearization of $\left(\mathbb{N}^{n}, \leq_{\text {pr }}\right)$ into $\left(\mathbb{N}^{n}, \leq_{\text {lex }}\right)$. As a side result, in Prop. 3 we obtain a tight upper bound for the length of the longest decreasing sequence in $\left(\mathbb{N}, \leq_{\text {lex }}\right)$.

Majoring and multiset ordering. Informally, if $A$ and $B$ are finite subsets of $\mathbb{N}^{n}$ then $A \leq_{\text {maj }} B$ iff every element of $A$ is majorized (with respect to $\leq_{\text {pr }}$ ) in $B$. It is well-known that $\leq_{\text {maj }}$ over subsets of $\mathbb{N}^{n}$ is a wqo, and this fact is used in a number of decidability results.

In Cor. 19 we show an upper bound for $L_{n, g}^{\text {maj }}(t)$, the length of the longest

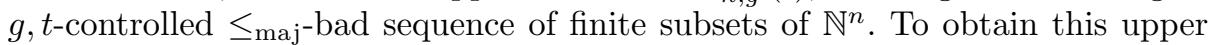
bound, we linearize the wqo into the multiset ordering over $\left(\mathbb{N}^{n}, \leq_{\text {lex }}\right)$, which is a well-order. In Thm. 12 and Thm. 14 we show a tight upper bound for the longest decreasing sequence of multisets.

We also give some applications on how our upper bound for $L_{n, g}^{\operatorname{maj}}(t)$ can be used in some decision procedures of some types of automata over data trees. 


\section{Outline}

In $\$ 2$ we give the formal definitions of all the involved orders and the definition of the Fast Growing Hierarchy. In $\$ 3, \$ 4 \$ 56$ we study the lexicographic, product, multiset and majoring ordering, respectively. In $\$ 7$ we mention some applications of our upper bounds in concrete decision procedures. We close with some conclusions and future work in $\$ 8$

\section{Basic definitions}

If $A$ is a set then $|A|$ denotes the cardinality of $A$. If $x \in A^{n}$ then the $i$-th coordinate of $x$ is denoted $x[i]$, so $x=\langle x[1], \ldots, x[n]\rangle$. Sequences are always in boldface and if $\mathbf{x}$ is a finite sequence then $|\mathbf{x}|$ denotes its length. The concatenation of the sequence $\mathbf{x}$ and the element $x$ at the rightmost place is denoted $\mathbf{x}^{\wedge} x$. We fix $g: \mathbb{N} \rightarrow \mathbb{N}$ to be a computable and increasing function.

Given a set $X$ provided with a total order $\leq,(X, \leq)$ is called a well-order if every non-empty subset of $X$ has a minimum.

We work with the following wqo's:

Lexicographic ordering. If $x, y \in \mathbb{N}^{n}$ then it is the well-order defined as

$$
x<_{\operatorname{lex}} y \stackrel{\text { def }}{\Leftrightarrow} x[1]<y[1] \vee\left(x[1]=y[1] \wedge\langle x[2], \ldots, x[n]\rangle<_{\operatorname{lex}}\langle y[2], \ldots, y[n]\rangle\right) .
$$

Product ordering. If $x, y \in \mathbb{N}^{n}$ then it is the wqo defined as

$$
x \leq_{\text {pr }} y \stackrel{\text { def }}{\Leftrightarrow}(\forall i \in\{1, \ldots, n\}) x[i] \leq y[i] .
$$

Multiset ordering. A multiset $M$ over a set $X$ is a function $X \rightarrow \mathbb{N}$. Intuitively a multiset is a generalization of a set, where elements may be repeated. For $x \in X$, $M(x)$ is called the multiplicity of $x$. A multiset is finite if the set of elements with positive multiplicity is finite. We notate $x \in M$ for $M(x)>0$. Let $\mathcal{M}_{<\infty}(X)$ denote the class of finite multisets over $X$.

Let $(X, \leq)$ be a poset and let $M, N \in \mathcal{M}_{<\infty}(X)$. We define

$N<\stackrel{(\leq)}{\mathrm{ms}} M \stackrel{\text { lef }}{\Leftrightarrow} M \neq N \wedge(\forall x \in X)[N(x)>M(x) \Rightarrow(\exists y \in X)[y>x \wedge M(y)>N(y)]]$.

Intuitively, this says that $N$ can be obtained from $M$ by replacing some elements by finitely many (possibly zero) smaller (with respect to $\leq$ ) elements. If $(X, \leq)$ is a well-order then $\left(\mathcal{M}_{<\infty}(X), \leq_{\mathrm{ms}}^{(\leq)}\right)$is also a well-order. See [3] for more details.

We will study $\left(\mathcal{M}_{<\infty}\left(\mathbb{N}^{n}\right), \leq_{\mathrm{ms}}^{\left(\leq_{\text {lex }}\right)}\right)$, the multiset ordering of finite multisets of tuples with the underlying lexicographic ordering. In this context, we write $\leq_{\mathrm{ms}}$ for $\leq_{\mathrm{ms}}^{\left(\leq_{\text {lex }}\right)}$. Observe that it is a well-order because $\left(\mathbb{N}^{n}, \leq_{\text {lex }}\right)$ is so.

Majoring ordering. Let $\mathcal{P}_{<\infty}(X)$ denote the finite and non-empty parts of $X$. For a wqo $(X, \leq)$ and $A, B \in \mathcal{P}_{<\infty}(X)$, the majoring ordering is defined as

$$
A \leq \underset{\text { maj }}{(\leq)} B \stackrel{\text { def }}{\Leftrightarrow}(\forall x \in A)(\exists y \in B) x \leq y .
$$


We will study $\left(\mathcal{P}<\infty\left(\mathbb{N}^{n}\right), \leq_{\text {maj }}^{\left(\leq_{\text {pr }}\right)}\right)$, the majoring ordering of finite sets of tuples with the underlying product ordering. In this context, we write $\leq_{\text {maj }}$ for $\leq_{\text {maj }}^{\left(\leq_{\text {p }}\right)}$. Observe that it is a wqo because $\left(\mathbb{N}^{n}, \leq_{\text {pr }}\right)$ is so (see for instance [4, Prop. 2.5]).

The Fast Growing Hierarchy $\left(F_{\alpha}\right)_{\alpha<\epsilon_{0}}$. Let $\epsilon_{0}$ be the least infinite ordinal $\alpha$ such that $\omega^{\alpha}=\alpha$. The Fast Growing Hierarchy is defined as

$$
F_{0}(x) \stackrel{\text { def }}{=} x+1 \quad F_{\alpha+1}(x) \stackrel{\text { def }}{=} F_{\alpha}^{x+1}(x) \quad F_{\lambda} \stackrel{\text { def }}{=} F_{\lambda_{x}}(x),
$$

where in general $g^{k}$ denotes the $k$-th iteration of $g$ (i.e. $g^{1}=g$ and $g^{k+1}=g \circ g^{k}$ ), $\alpha<\epsilon_{0}$ is an ordinal, $\lambda<\epsilon_{0}$ is a limit ordinal and $\left(\lambda_{x}\right)_{x<\omega}$ is an increasing sequence of ordinals with limit $\lambda$ (a fundamental sequence), which we fix to be:

$$
\left(\gamma+\omega^{\beta+1}\right)_{x} \stackrel{\text { def }}{=} \gamma+\omega^{\beta} \cdot(x+1) \quad\left(\gamma+\omega^{\lambda}\right)_{x} \stackrel{\text { def }}{=} \gamma+\omega^{\lambda_{x}} .
$$

The class $\mathfrak{F}_{\alpha}$ of the Fast Growing Hierarchy is the closure under substitution and limited recursion of the constant, sum, projections, and the functions $F_{\alpha}$. $\mathfrak{F}_{0}=\mathfrak{F}_{1}$ contains all linear functions, $\mathfrak{F}_{2}$ contains all the elementary functions, $\mathfrak{F}_{3}$ contains all the tetration functions. $\bigcup_{n<\omega} \mathfrak{F}_{n}$ is the class of all primitive recursive functions and in general $\bigcup_{\alpha<\omega^{k}} \mathfrak{F}_{\alpha}$ is the class of $k$-recursive functions [12. There are a number of important monotonicity results regarding the Fast Growing Hierarchy: for ordinals $\alpha<\beta<\epsilon_{0}$, the function $F_{\alpha}$ is strictly increasing, $F_{\alpha+1} \geq$ $F_{\alpha}, F_{\alpha}$ is eventually majorized by $F_{\beta}$, and then $\mathfrak{F}_{\alpha} \subsetneq \mathfrak{F}_{\beta}$ (except $\alpha=0$ and $\beta=1$ ), etc. For more results on the Fast Growing Hierarchy, cf. [10].

\section{The lexicographic ordering}

We denote by $L_{n, g}^{\text {lex }}(t)$ the length of the longest $g, t$-controlled decreasing sequence in $\left(\mathbb{N}^{n}, \leq_{\text {lex }}\right)$. In [5, Section VI], it is shown that

$$
L_{1, g}^{\text {lex }}(t)=g(t), \quad L_{n+1, g}^{\text {lex }}(t)=\sum_{j=1}^{g(t)} L_{n, g}^{\text {lex }}\left(o_{n, g}^{j-1}(t)\right), \quad o_{n, g}(t) \stackrel{\text { def }}{=} t+L_{n, g}^{\text {lex }}(t) .
$$

Proposition 3. For any ordinal $\gamma \geq 1$, if $g \in \mathfrak{F}_{\gamma}$ then $L_{n, g}^{\text {lex }}$ has an upper bound in $\mathfrak{F}_{\gamma+n-1}$.

Proof. We proceed by induction on $n$. If $n=1$ then $L_{1, g}^{\text {lex }}(t)=g(t)$, and by hypothesis $g \in \mathfrak{F}_{\gamma}$. Now suppose $L_{n, g}^{\text {lex }} \leq h \in \mathfrak{F}_{\gamma+n-1}$. We have $L_{n+1, g}^{\text {lex }}(t) \leq$ $g(t) \cdot L_{n, g}^{\text {lex }}\left(o_{n, g}^{g(t)-1}(t)\right) \leq g(t) \cdot o_{n, g}^{g(t)}(t)$ where the first inequality follows from 3 , since $o_{n, g}$ is growing, and the second one because $L_{n, g}^{\text {lex }} \leq o_{n, g}$.

Since $L_{n, g}^{\text {lex }} \leq h \in \mathfrak{F}_{\gamma+n-1}$ then $o_{n, g}(t) \leq h(t)+t$ and so $o_{n, g} \in \mathfrak{F}_{\gamma+n-1}$. By [10, Thm. 2.10], there is $p$ such that $F_{\gamma+n-1}^{p}$ majorizes $o_{n, g}$. Therefore

$$
\begin{aligned}
L_{n+1, g}^{\text {lex }}(t) & <g(t) \cdot F_{\gamma+n-1}^{p \cdot g(t)}(t) \\
& \left.\leq g(t) \cdot F_{\gamma+n-1}^{p \cdot g(t)+1}(p \cdot g(t)) \quad \text { (by monononicity of } F_{\gamma+n-1}\right) \\
& =g(t) \cdot F_{\gamma+n}(p \cdot g(t)),
\end{aligned}
$$


which lies in $\mathfrak{F}_{\gamma+n}$, since it is the composition and product of functions in $\mathfrak{F}_{\gamma+n}$ (and since $\gamma+n \geq 2, \mathfrak{F}_{\gamma+n}$ is closed by products).

In [5, Prop. VI.3] it is shown that if $g=F_{\gamma}$ then $L_{n, g}^{\text {lex }} \geq F_{\gamma+n-1}$. Hence our upper bound is tight.

\section{The product ordering}

In this section we linearize the wqo $\left(\mathbb{N}^{n}, \leq_{\mathrm{pr}}\right)$ into the well-order $\left(\mathbb{N}^{n}, \leq_{\text {lex }}\right)$ and derive an upper bound for $L_{n, g}^{\mathrm{pr}}(t)$, the length of the longest $g, t$-controlled bad sequence over $\left(\mathbb{N}^{n}, \leq_{\mathrm{pr}}\right)$.

The next result follows the idea of Harwood, Moller and Setzer [7] adapted to controlled bad sequences. For the sake of completeness we include the full proof.

First, let us mention the intuition behind the proof. For $x \in \mathbb{N}^{n}$, define $\uparrow x \stackrel{\text { def }}{=}\left\{z \in \mathbb{N}^{n} \mid x \leq_{\mathrm{pr}} z\right\}$. Let $n=2$, and suppose

$$
\mathbf{x}=\left\langle x_{0}, y_{0}\right\rangle,\left\langle x_{1}, y_{1}\right\rangle,\left\langle x_{2}, y_{2}\right\rangle, \ldots,\left\langle x_{k}, y_{k}\right\rangle
$$

is a bad sequence in $\left(\mathbb{N}^{2}, \leq_{\mathrm{pr}}\right)$. Let $a(\mathbf{x})=\min _{0 \leq i<|\mathbf{x}|} x_{i}, b(\mathbf{x})=\min _{0 \leq i<|\mathbf{x}|} y_{i}$ and $C(\mathbf{x})=\uparrow\langle a(\mathbf{x}), b(\mathbf{x})\rangle \backslash \bigcup_{0 \leq i<|\mathbf{x}|} \uparrow\left\langle x_{i}, y_{i}\right\rangle$. It is easy to see that $C(\mathbf{x})$ is finite. Here is how we can linearize $\left(\mathbb{N}^{2}, \leq_{\text {pr }}\right)$ into $\left(\mathbb{N}^{2}, \leq_{\text {lex }}\right)$ : Define $h(\mathbf{x}) \stackrel{\text { def }}{=}\langle a(\mathbf{x})+$ $b(\mathbf{x}),|C(\mathbf{x})|\rangle \in \mathbb{N}^{2}$ and suppose that $\mathbf{x}^{\wedge}\langle x, y\rangle$ is bad. If $x<a(\mathbf{x}) \vee y<b(\mathbf{x})$ then $h\left(\mathbf{x}^{\wedge}\langle x, y\rangle\right)[1]<h(\mathbf{x})[1]$; in case $x \geq a(\mathbf{x}) \wedge y \geq b(\mathbf{x})$ then $C\left(\mathbf{x}^{\wedge}\langle x, y\rangle\right) \subseteq C(\mathbf{x})$. In this last case, since $\langle x, y\rangle \in C(\mathbf{x}) \backslash C\left(\mathbf{x}^{\wedge}\langle x, y\rangle\right)$, we have $\left|C\left(\mathbf{x}^{\wedge}\langle x, y\rangle\right)\right|<|C(\mathbf{x})|$. Therefore $h\left(\mathbf{x}^{\wedge}\langle x, y\rangle\right)<_{\text {lex }} h(\mathbf{x})$. Furthermore, if $\mathbf{x}$ is $g, t$-controlled then $C(\mathbf{x})$ has at most $g(t+|\mathbf{x}|-1)^{2}$ elements, and $a(\mathbf{x})+b(\mathbf{x})<2 g(t+|\mathbf{x}|-1)$. Hence if $\mathbf{x}$ is $g, t$-controlled, then the sequence

$$
\mathbf{y}=h\left(\left\langle x_{0}, y_{0}\right\rangle\right), h\left(\left\langle x_{0}, y_{0}\right\rangle,\left\langle x_{1}, y_{1}\right\rangle\right), \ldots, h(\mathbf{x}),
$$

is $<_{\text {lex }}$-descending and $\tilde{g}, t$-controlled, where $\tilde{g}(x)=2 g(x)^{2}$.

The argument for any $n>2$ cannot be generalized straightforwardly, obtaining a linearization into $\left(\mathbb{N}^{2}, \leq_{\text {lex }}\right)$. For instance, for $n=3$ and $\mathbf{x}=\langle 0,0,1\rangle,\langle 0,1,0\rangle$, we would have $C(\mathbf{x})=\uparrow\langle 0,0,0\rangle \backslash(\uparrow\langle 0,0,1\rangle \cup \uparrow\langle 0,1,0\rangle)$ and this set is infinite $((N, 0,0) \in C(\mathbf{x})$ for any $N)$. However, by an inductive argument $\left(\mathbb{N}^{n}, \leq_{\text {pr }}\right)$ can be linearized into $\left(\mathbb{N}^{n}, \leq_{\text {lex }}\right)$.

Theorem 4. There is a function $h_{n}:\left(\mathbb{N}^{n}\right)^{+} \rightarrow \mathbb{N}^{n}$ such that if $\mathbf{x}^{\frown} x$ is bad in $\left(\mathbb{N}^{n}, \leq_{\mathrm{pr}}\right)$ and $\mathbf{x}$ is nonempty, then $h_{n}\left(\mathbf{x}^{-} x\right)<_{\operatorname{lex}} h_{n}(\mathbf{x})$. Furthermore if $\mathbf{x}$ is $g, t$-controlled then $\left|h_{n}(\mathbf{x})\right|_{\infty}<\tilde{g}(|\mathbf{x}|-1+t)$, for $\tilde{g}(x)=n ! g(n x)^{n}$.

Proof. We define the functions $h_{n}$ by induction in $n$. If $\mathbf{x}=x_{0}, x_{1}, x_{2}, \ldots, x_{k}$ is a bad sequence in $\mathbb{N}$ then define $h_{1}\left(x_{0}, x_{1}, x_{2}, \ldots, x_{k}\right) \stackrel{\text { def }}{=} x_{k}$. Since in $\mathbb{N}$ the product order and the lexicographic order coincide, we have $h_{1}\left(\mathbf{x}^{\wedge} x\right)<_{\operatorname{lex}} h_{1}(\mathbf{x})$.

For the inductive construction of $h_{n}$, let $n>1$ and assume the statement of the theorem for dimension $n-1$. For $1 \leq i \leq n$ and $x \in \mathbb{N}^{n}$ we define

$$
\operatorname{DEL}_{i}(x) \stackrel{\text { def }}{=}\langle x[1], \ldots, x[i-1], x[i+1], \ldots, x[n]\rangle,
$$


i.e. $\operatorname{DEL}_{i}(x)$ deletes the $i$-th component of the $n$-tuple $x$. Given a finite and nonempty bad sequence $\mathbf{x}=x_{0}, x_{1}, \ldots, x_{k}$ of $n$-tuples, we define the set

$$
\begin{aligned}
& \operatorname{BAD}_{i}(\mathbf{x}) \stackrel{\text { def }}{=}\left\{\operatorname{DEL}_{i}\left(x_{j_{0}}\right), \ldots, \operatorname{DEL}_{i}\left(x_{j_{p}}\right) \mid p \geq 0,0 \leq j_{0}<\cdots<j_{p} \leq k\right. \text {, and } \\
& \operatorname{DEL}_{i}\left(x_{j_{0}}\right), \ldots, \operatorname{DEL}_{i}\left(x_{j_{p}}\right) \text { is bad\}, }
\end{aligned}
$$

i.e. $\operatorname{BAD}_{i}(\mathbf{x})$ consists of the bad subsequences of $(n-1)$-tuples of $\mathbf{x}$ in which the $i$-th components of the $n$-tuples have been deleted. Finally we define

$$
\begin{gathered}
\operatorname{MIN}_{i}(\mathbf{x}) \stackrel{\text { def }}{=} \min _{<\text {lex }}\left\{h_{n-1}(\mathbf{y}) \mid \mathbf{y} \in \operatorname{BAD}_{i}(\mathbf{x})\right\} \quad \text { and } \\
\operatorname{EXT}_{n}(\mathbf{x}) \stackrel{\text { def }}{=}\left\{x \in \mathbb{N}^{n} \mid(\forall i \in\{1, \ldots, n\}) \operatorname{MIN}_{i}(\mathbf{x})=\operatorname{MIN}_{i}\left(\mathbf{x}^{\frown} x\right),\right. \text { and } \\
\left.(\forall j \in\{0, \ldots, k\}) x_{j} \not \mathbb{p}_{\mathrm{pr}} x\right\}
\end{gathered}
$$

which consists of the $n$-tuples with which the sequence $\mathbf{x}$ can be extended without altering the $\operatorname{MIN}_{i}$ values and yet while maintaining badness.

Fact $1\left|\operatorname{EXT}_{n}(\mathbf{x})\right|<\infty$, and if $\mathbf{x}$ is $g$, t-controlled, then $\left|\operatorname{EXT}_{n}(\mathbf{x})\right|<g(k+t)^{n}$.

Proof. Let $\mathbf{z}=\operatorname{DEL}_{i}\left(x_{j_{0}}\right), \ldots, \operatorname{DEL}_{i}\left(x_{j_{p}}\right) \in\left(\mathbb{N}^{n-1}\right)^{+}$be a bad sequence, suppose $\operatorname{MIN}_{i}(\mathbf{x})=h_{n-1}(\mathbf{z})$, and suppose that $s \in \operatorname{EXT}_{n}(\mathbf{x})$. If the sequence $\mathbf{z}^{\frown} \operatorname{DEL}_{i}(s)$ were bad, then by the ind. hyp. we would get that $\operatorname{MIN}_{i}\left(\mathbf{x}^{\urcorner} s\right) \leq_{\text {lex }} h_{n-1}\left(\mathbf{z}^{\frown} \operatorname{DEL}_{i}(s)\right)<_{\text {lex }}$ $h_{n-1}(\mathbf{z})=\operatorname{MIN}_{i}(\mathbf{x})$, contradicting $s \in \operatorname{EXT}_{n}(\mathbf{x})$. Therefore, since $\mathbf{z}$ is bad but $\mathbf{z}^{\frown} \operatorname{DEL}_{i}(s)$ is not, we have $\operatorname{DEL}_{i}\left(x_{j_{m}}\right) \leq_{\text {pr }} \operatorname{DEL}_{i}(s)$ for some $m$. But since $s \in$ $\operatorname{EXT}_{n}(\mathbf{x})$ we have that $x_{j_{m}} \underline{\text { pr }}_{\mathrm{pr}} s$, and therefore $s[i]<x_{j_{m}}[i]$. Now, since this goes for all $i$, we conclude that $\left|\operatorname{ExT}_{n}(\mathbf{x})\right|$ is finite.

Now if $\mathbf{x}$ is $g, t$-controlled, then $x_{j}[i]<g(k+t)$ for all $j$, because $g$ is increasing. By the above argument $\left|\operatorname{EXT}_{n}(\mathbf{x})\right| \leq g(k+t)^{n}$, but since $\mathbf{x}$ was nonempty and $x_{0} \notin \operatorname{EXT}_{n}(\mathbf{x})$, we conclude $\left|\operatorname{EXT}_{n}(\mathbf{x})\right|<g(k+t)^{n}$.

We finally define

$$
h_{n}(\mathbf{x}) \stackrel{\text { def }}{=}\left\langle\sum_{i=1}^{n} \operatorname{MIN}_{i}(\mathbf{x}),\left|\operatorname{EXT}_{n}(\mathbf{x})\right|\right\rangle \in \mathbb{N}^{n},
$$

where the sum is taken componentwise and thus results in a tuple in $\mathbb{N}^{n-1}$. We conclude the proof with the following two facts:

Fact 2 If $\mathbf{x}^{\frown} x$ is bad then $h_{n}\left(\mathbf{x}^{\frown} x\right)<_{\text {lex }} h_{n}(\mathbf{x})$.

Proof. Suppose that $\mathbf{y}=\mathbf{x}^{\frown} x$ bad. Since for any $i \in\{1, \ldots, n\}, \operatorname{BAD}_{i}(\mathbf{x}) \subseteq$ $\operatorname{BAD}_{i}(\mathbf{y})$, then $\operatorname{MIN}_{i}(\mathbf{y}) \leq_{\text {lex }} \operatorname{MIN}_{i}(\mathbf{x})$; and if $\operatorname{MIN}_{i}(\mathbf{y})=\operatorname{MIN}_{i}(\mathbf{x})$ for all $i$ then $\operatorname{EXT}_{n}(\mathbf{y}) \subsetneq \operatorname{EXT}_{n}(\mathbf{x})$, since $\operatorname{EXT}_{n}(\mathbf{y}) \subseteq \operatorname{EXT}_{n}(\mathbf{x})$ but $x \in \operatorname{EXT}_{n}(\mathbf{x}) \backslash \operatorname{EXT}_{n}(\mathbf{y})$. Thus $\left|\operatorname{EXT}_{n}(\mathbf{y})\right|<\left|\operatorname{EXT}_{n}(\mathbf{x})\right|$.

Fact 3 If $\mathbf{x}$ is $g, t$ controlled then $\left|h_{n}(\mathbf{x})\right|_{\infty}<\tilde{g}(|\mathbf{x}|-1+t)$, where $\tilde{g}(x)=$ $n ! g(n x)^{n}$. 
Proof. By induction in $n \geq 1$. If $n=1$ then If $\mathbf{x}=x_{0}, \ldots, x_{k}$ is $g, t$-controlled, then $h_{1} \mathbf{x}=x_{k}<g(t+k)=g(t+|\mathbf{x}|-1)=\tilde{g}(t+|\mathbf{x}|-1)$.

Since any $\mathbf{y} \in \operatorname{BAD}_{i}(\mathbf{x})$ is a $g,(t+k)$-controlled bad sequence of $\mathbb{N}^{n-1}$, by inductive hypothesis we get

$$
\begin{aligned}
\left|h_{n-1}(\mathbf{y})\right|_{\infty} & <(n-1) ! g((n-1)(|\mathbf{y}|-1)+t+k)^{n-1} \\
& \leq(n-1) ! g((n-1) k+t+k)^{n-1} \\
& =(n-1) ! g(n k+t)^{n-1} .
\end{aligned}
$$

In particular, for $\mathbf{y}$ such that $\operatorname{MIN}_{i}(\mathbf{x})=h_{n-1}(\mathbf{y})$, we conclude $\left|\operatorname{MIN}_{i}(\mathbf{x})\right|_{\infty}<$ $(n-1) ! g(n k+t)^{n-1}$, and so the first $n-1$ coordinates of $h_{n}(\mathbf{x})$ are strictly bounded by $n ! g(n k+t)^{n-1}$ (the factor $n$ comes from the $n$ additions). By Fact 1 the last coordinate of $h_{n}(\mathbf{x})$ is strictly bounded by $g(k+t)^{n}$. Therefore, $\left|h_{n}(\mathbf{x})\right|_{\infty}<\max \left\{n ! g(n k+t)^{n-1}, g(k+t)^{n}\right\} \leq n ! g(n k+t)^{n} \leq \tilde{g}(|\mathbf{x}|-1+t)$.

Let $L_{n, g}^{\mathrm{pr}}(t)$ denote the length of the longest $g, t$-controlled bad sequence in $\left(\mathbb{N}^{n}, \leq_{\mathrm{pr}}\right)$, and let $L_{n, g}^{\text {lex }}(t)$ denote the length of the longest $g, t$-controlled decreasing sequence in $\left(\mathbb{N}^{n}, \leq_{\text {lex }}\right)$. We arrive to the same result as in [5]:

Corollary 5. $L_{n, g}^{\mathrm{pr}} \leq L_{n, \tilde{g}}^{\mathrm{lex}}$, for $\tilde{g}$ as in Thm. 4 Hence if $g \in \mathfrak{F}_{\gamma}$, and $\gamma \geq 2$ is an ordinal, then $L_{n, g}^{\mathrm{pr}}$ has an upper bound in $\mathfrak{F}_{\gamma+n-1}$.

Proof. The function $\tilde{g}$ is defined through finite substitution from $g$ and product. Since $\mathfrak{F}_{2}$ and higher levels are closed under finite products, we have $\tilde{g} \in \mathfrak{F}_{\gamma}$. By Prop. 3. there is a function $h \in \mathfrak{F}_{\gamma+n-1}$ such that $h \geq L_{n, \tilde{g}}^{\text {lex }}$.

\section{The multiset ordering}

We need a notion of $g, t$-controlled sequence of (multi)sets. By Def. 2 it suffices to give a proper norm:

Definition 6 (A proper norm of sets and multisets of tuples). Given $X \in \mathcal{M}_{<\infty}\left(\mathbb{N}^{n}\right)$, we define $|X|$, the norm of $X$, as the maximum between $\max _{x \in \mathbb{N}^{n}} X(x)$ and $\max \left\{|x|_{\infty} \mid x \in \mathbb{N}^{n} \wedge X(x)>0\right\}$. For $X \in \mathcal{P}_{<\infty}\left(\mathbb{N}^{n}\right),|X|$ is defined analogously, as any set is a multiset.

We denote by $L_{g, n}^{\mathrm{ms}}(t)$ the length of the longest $g, t$-controlled decreasing sequence in $\left(\mathcal{M}_{<\infty}\left(\mathbb{N}^{n}\right), \leq_{\text {ms }}^{\left(\leq_{\text {lex }}\right)}\right)$, i.e. a sequence of finite multisets of $\mathbb{N}^{n}$, with the underlying lexicographic ordering. In this section we give a tight upper bound for $L_{g, n}^{\mathrm{ms}}(t)$ in terms of the Fast Growing Hierarchy.

\subsection{Maximizing strategy.}

To study the longest $g, t$-controlled $\leq_{\mathrm{ms}}$-descending sequence of multisets we define the maximizing strategy which, given a nonempty $g, t$-controlled multiset $M$, determines the greatest $g,(t+1)$-controlled multiset $N$ which is smaller than 
$M$. The strategy says that to obtain $N$ one should take out one of the minimum elements of $M$, say $m$, (i.e. decrement in one the multiplicity of $m$ ) and add as many elements smaller than $m$ as the control function permits.

For the rest of this subsection, assume $(X, \leq)$ is a well-order. We write $<_{\mathrm{ms}}$ instead of $<_{\mathrm{ms}}^{(\leq)}$. Let $M \in \mathcal{M}_{<\infty}(X)$ which is $g, t$-controlled and a proper norm $|\cdot|_{X}=|\cdot|$ for $X$. We define the $g, t$-predecessor of $M$ as follows: For $x \in X$,

$$
\operatorname{PRED}_{t}^{g}(M)(x) \stackrel{\text { def }}{=} \begin{cases}g(t+1)-1 & x<\min M \wedge|x|<g(t+1) ; \\ M(x)-1 & x=\min M \\ M(x) & \text { otherwise. }\end{cases}
$$

where $\min M \stackrel{\text { def }}{=} \min \{x \mid M(x)>0\}$.

Lemma 7. Let $M$ be a nonempty finite multiset over a totally ordered set $P$, which is $g$,t-controlled and let $N=\operatorname{PRED}_{t}^{g}(M)$. Then (1) $N$ is $g,(t+1)$ controlled; (2) $N<_{\mathrm{ms}} M$; and (3) if $N^{\prime}$ is $g,(t+1)$-controlled and $N^{\prime}<_{\mathrm{ms}} M$ then $N^{\prime} \leq_{\mathrm{ms}} N$.

Proof. (1) is clear from the definition of $N$ and the fact that $g$ is monotone increasing. For (2), it is obvious that $M \neq N$. By definition, if $N(x)>M(x)$ then $x<m=\min M$ and $M(m)>N(m)$.

For (3), assume $N^{\prime}<M$ is $g,(t+1)$-controlled. We show that if $N^{\prime}(x)>N(x)$ then there is $z>x$ such that $N(z)>N^{\prime}(z)$. Suppose $N^{\prime}(x)>N(x)$. First, if $x<\min M$ then $N(x)=g(t+1)-1 \geq N^{\prime}(x)$, contradicting $N^{\prime}(x)>N(x)$. Second, suppose $x>\min M$. Then $N(x)=M(x)$ and therefore $N^{\prime}(x)>M(x)$. Since $N^{\prime}<_{\mathrm{ms}} M$ there is $z>x$ such that $N(z)=M(z)>N^{\prime}(z)$. Third, suppose $x=\min M$. Then $N(x)=M(x)-1$, and so $N^{\prime}(x) \geq M(x)$. If $N^{\prime}(x)>M(x)$ then, since $M<_{\mathrm{ms}} N^{\prime}$, there is $z>x$ with $M(z)>N^{\prime}(z)$. For such $z$, by definition of $N$, we have $N(z)=M(z)>N^{\prime}(z)$. If $N^{\prime}(x)=M(x)$ then, since $N^{\prime} \neq M$, there is $y$ such that $N^{\prime}(y) \neq M(y)$. Any such $y$ must be different from $x$. Suppose that all such $y^{\prime}$ 's were smaller than $x=\min M$. In this case $M \leq_{\mathrm{ms}} N^{\prime}$ and this contradicts the hypothesis. Hence there is $y>x$ such that $N^{\prime}(y) \neq M(y)$. If $N^{\prime}(y)>M(y)$, there is $z>y>x$ such that $N^{\prime}(z)<M(z)=$ $N(z)$. If $N^{\prime}(y)<M(y)$, since $M(y)=N(y)$, we conclude $N^{\prime}(y)<N(y)$.

We represent a finite multiset $M$ such that $\{x \mid M(x)>0\}=\left\{x_{1}, \ldots, x_{n}\right\}$ as $M \stackrel{\text { def }}{=} M\left(x_{1}\right) \cdot x_{1}+\cdots+M\left(x_{n}\right) \cdot x_{n}$.

For a finite multiset $M$, let $L_{g, M}(t)$ denote the length minus one of the longest $g, t$-controlled and $<_{\mathrm{ms}}$-decreasing sequence of multisets starting with the multiset $M$. For $x \in X$, let $o_{g, x}(t)=t+L_{g, 1 \cdot\{x\}}(t)$.

Lemma 8. If $k \geq 1$ then $L_{g, k \cdot\{x\}}(t)=\sum_{i=0}^{k-1} L_{g, 1 \cdot\{x\}}\left(o_{g, x}^{i}(t)\right)$.

Proof (Sketch). We write $L_{k}$ for $L_{g, k} \cdot\{x\}$ and $o$ for $o_{g, x}$. First show by induction in $i$ that $o^{i}(t)=t+\sum_{j=0}^{i-1} L_{1}\left(o^{j}(t)\right)$. Then show the statement of the Lemma by 
induction in $k \geq 1$. Observe that the longest $g, t$-controlled decreasing sequence of multisets beginning with $M_{1}=(k+1) \cdot\{x\}$ is

$$
M_{1}>_{\mathrm{ms}} M_{2}>_{\mathrm{ms}} \ldots,>_{\mathrm{ms}} M_{l_{1}}>_{\mathrm{ms}} N_{2}>_{\mathrm{ms}} N_{3}>_{\mathrm{ms}} \ldots>_{\mathrm{ms}} N_{l_{2}},
$$

of length $l_{1}+l_{2}-1$ and where $l_{1}=L_{k}(t)+1, M_{l_{1}}=1 \cdot\{x\}, l_{2}=L_{1}\left(t+L_{k}(t)\right)+1$ and $N_{l_{2}}=\emptyset$. Use straightforwardly the inductive hypothesis.

Corollary 9. For $k \geq 1, L_{g, k \cdot\{x\}} \geq L_{g, 1 \cdot\{x\}}^{k}$.

Corollary 10. For $k \geq 1, L_{g, k \cdot\{x\}}(t) \leq k \cdot L_{g, 1 \cdot\{x\}}\left(o_{g, x}^{k-1}(t)\right)$.

In the sequel we fix $(X, \leq)$ to be $\left(\mathbb{N}^{n}, \leq_{\text {lex }}\right)$. If $M \in \mathcal{M}_{<\infty}\left(\mathbb{N}^{n}\right)$ then $P_{g, n}(M, t)$ denotes the length minus one of the longest $g, t$-controlled $<_{\mathrm{ms}^{-}}$-decreasing sequence of multisets starting with $M$. If $M$ consists of one copy of $\left(x_{1}, \ldots, x_{n}\right)$, we simply write $P_{g, n}\left(x_{1}, \ldots, x_{n}, t\right)$ instead of $P_{g, n}\left(1 \cdot\left\{\left(x_{1}, \ldots, x_{n}\right)\right\}, t\right)$. Observe that, having fixed $(X, \leq)$, we have $L_{g, M}(t)=P_{g, n}(M, t)$.

\subsection{Lower bound.}

Define $G_{g, n}: \mathbb{N}^{n+1} \backslash\{(0, \ldots, 0)\} \rightarrow \mathbb{N}$ by multiple recursion as:

$$
\begin{aligned}
G_{g, n}(0, \ldots, 0,1, t) & \stackrel{\text { def }}{=} g(t+1) \\
G_{g, n}\left(\bar{x}, x_{n}+1, t\right) & \stackrel{\text { def }}{=} G_{g, n}^{g(t+1)-1}\left(\bar{x}, x_{n}, t\right), \text { for } \bar{x}=x_{1}, \ldots, x_{n-1} \\
G_{g, n}\left(\bar{x}, x_{j}+1, \overline{0}, t\right) & \stackrel{\text { def }}{=} G_{g, n}\left(\bar{x}, x_{j}, g(t+1)-1, \overline{0}, t\right), \text { for } \bar{x}=x_{1}, \ldots, x_{j-1}
\end{aligned}
$$

Equation (5) applies when $x_{i}>0$ for some $i$, and (6) when $j<n . G_{g, n}^{k}(\bar{a}, b)$ denotes the $k$-th iteration of $G_{g, n}$ in the last component, i.e. $G_{g, n}^{1}(\bar{a}, b) \stackrel{g, n}{=} G_{g, n}(\bar{a}, b)$ and $G_{g, n}^{k+1}(\bar{a}, b)=G_{g, n}\left(\bar{a}, G_{g, n}^{k}(\bar{a}, b)\right)$.

Lemma 11. If $g(x) \geq x+1$ then $P_{g, n} \geq G_{g, n}$.

Proof (Sketch). By induction in the lexicographic order of $x_{1}, \ldots, x_{n}$. For (4), the longest $g, t$-controlled $<_{\mathrm{ms}^{-}}$-decreasing sequence starting with $1 \cdot\{(\overline{0}, 1)\}$ is

$$
1 \cdot\{(\overline{0}, 1)\}>_{\mathrm{ms}}(g(t+1)-1) \cdot\{(\overline{0}, 0)\}>_{\mathrm{ms}} \ldots>_{\mathrm{ms}} 0 \cdot\{(\overline{0}, 0)\}=\emptyset,
$$

which has length $g(t+1)+1$ and then $P_{g, n}(0, \ldots, 0,1, t)=g(t+1)$. For (5), the longest $g, t$-controlled $<_{\text {ms }}$-decreasing sequence of multisets starting with $1 \cdot\left\{\left(\bar{x}, x_{n}+1\right)\right\}$ contains the multiset $M=(g(t+1)-1) \cdot\left\{\left(\bar{x}, x_{n}\right)\right\}$, so $P_{g, n}\left(\bar{x}, x_{n}+\right.$ $1, t) \geq P_{g, n}(M, t+1)$. Now apply Cor. 9. monotonicity of $G_{g, n}$ and ind. hyp. For (6), the longest $g, t$-controlled $<_{\mathrm{ms}}$-decreasing sequence of multisets starting with $1 \cdot\left\{\left(\bar{x}, x_{j}+1, \overline{0}\right)\right\}$ contains $1 \cdot\left\{\left(x_{1}, \ldots, x_{j}, g(t+1)-1, \overline{0}\right)\right\}$ as one of its terms, so $P_{g, n}\left(\bar{x}, x_{j}+1, \overline{0}, t\right) \geq P_{g, n}\left(\bar{x}, x_{j}, g(t+1)-1, \overline{0}, t\right)$. Then apply ind. hyp.

Theorem 12. If $g \geq F_{1}$ and $g(x) \geq x+2$, then $L_{g, n}^{\mathrm{ms}} \geq F_{\omega^{n}}$. 
Proof (Sketch). Show that if $x_{i}>0$ for some $i$ then $G_{g, n}\left(x_{n-1}, \ldots, x_{0}, t\right) \geq$ $F_{\alpha}(t)$, where $\alpha=\omega^{n-1} \cdot x_{n-1}+\cdots+x_{0} \cdot \omega^{0}$ by induction in $\left(x_{n-1}, \ldots, x_{0}\right)$. Use monotonicity of $G_{g, n}$ and the fact that $g(x) \geq x+2$. Finally, for all $t$ we have

$$
\begin{aligned}
L_{g, n}^{\mathrm{ms}}(t) & \geq P_{g, n}(g(t)-1, \overline{0}, t) \\
& \geq P_{g, n}(t+1, \overline{0}, t) \\
& \geq G_{g, n}(t+1, \overline{0}, t) \\
& \geq F_{\omega^{n-1} \cdot(t+1)}(t)=F_{\omega^{n}}(t) .
\end{aligned}
$$

The second inequality follows from the monotonicity of $P_{g, n}$ and $g(x) \geq x+2$; the third one from Lem. 11 .

\subsection{Upper bound.}

Define $U_{g, n}: \mathbb{N}^{n+1} \backslash\{(0, \ldots, 0)\} \rightarrow \mathbb{N}$ by multiple recursion as:

$$
\begin{aligned}
U_{g, n}(0, \ldots, 0,1, t) & \stackrel{\stackrel{\text { def }}{=}}{=} g(t+1) \\
U_{g, n}\left(\bar{x}, x_{n}+1, t\right) & \stackrel{\text { def }}{=} g(t+1) \cdot U_{g, n}\left(\bar{x}, x_{n}, o_{x_{1}, \ldots, x_{n}}^{g(t+1)-1}(t+2)\right) \\
U_{g, n}\left(\bar{x}, x_{j}+1, \overline{0}, t\right) & \stackrel{\text { def }}{=} U_{g, n}\left(\bar{x}, x_{j}, g(t+1), \overline{0}, t+2\right)
\end{aligned}
$$

where $o_{x_{1}, \ldots, x_{n}}(t)=t+U_{g, n}\left(x_{1}, \ldots, x_{n-1}, x_{n}, t\right)$; equation (8) applies when $x_{i}>$ 0 and $\bar{x}=x_{1}, \ldots, x_{n-1}$; and equation (9) applies when $j<n$ and $\bar{x}=x_{1}, \ldots, x_{j-1}$.

Lemma 13. $P_{g, n} \leq U_{g, n}$.

Proof. By induction in the lexicographic order of $x_{1}, \ldots, x_{n}$. For (7), as in the proof of Lem. 11. the longest $g, t$-controlled $<_{\mathrm{ms}}$-decreasing sequence starting with $1 \cdot\{(\overline{0}, 1)\}$ has length $g(t+1)+1$ and then $P_{g, n}(\overline{0}, 1, t)=g(t+1)=$ $U_{g, n}(\overline{0}, 1, t)$. For $(8)$ the longest $g, t$-controlled $<_{\text {ms }}$-decreasing sequence starting with $M_{0}=1 \cdot\left\{\left(\bar{x}, x_{n}+1\right)\right\}$ continues with a multiset $M_{1}$ whose $<_{\text {lex }}$-maximum element is $\left(\bar{x}, x_{n}\right)$, of multiplicity $g(t+1)-1$. Therefore if $N=g(t+1) \cdot\left\{\left(\bar{x}, x_{n}\right)\right\}$ then $M_{0}>_{\mathrm{ms}} N>_{\mathrm{ms}} M_{1}$ and $N$ is $g,(t+2)$-controlled. Hence

$$
\begin{aligned}
P_{g, n}\left(\bar{x}, x_{n}+1, t\right) & \leq P_{g, n}\left(g(t+1) \cdot\left\{\left(\bar{x}, x_{n}\right)\right\}, t+2\right) \\
& \leq g(t+1) \cdot P_{g, n}\left(\bar{x}, x_{n}, \tilde{o}_{x_{1}, \ldots, x_{n}}^{g(t+1)-1}(t+2)\right) \\
& \leq g(t+1) \cdot U_{g, n}\left(\bar{x}, x_{n}, o_{x_{1}, \ldots, x_{n}}^{g(t+1)-1}(t+2)\right)=U_{g, n}\left(\bar{x}, x_{n}+1, t\right)
\end{aligned}
$$

where $\tilde{o}_{x_{1}, \ldots, x_{n}}(t)=t+P_{g, n}\left(x_{1}, \ldots, x_{n}, t\right)$, the second inequality follows from Cor. 10 and the third one from ind. hyp. and monotonicity of $U_{g, n}$. For 8 the longest $g, t$-controlled $<_{\mathrm{ms}}$-decreasing sequence of multisets starting with $M_{0}^{\prime}=1 \cdot\left\{\left(\bar{x}, x_{j}+1, \overline{0}\right)\right\}$ continues with a multiset $M_{1}^{\prime}$ whose $<_{\text {lex }}$-maximum element is $\left(\bar{x}, x_{j}, g(t+1)-1, \ldots, g(t+1)-1\right)$, of multiplicity $g(t+1)-1$. Then $M_{0}^{\prime}>_{\mathrm{ms}} N^{\prime}>_{\mathrm{ms}} M_{1}^{\prime}$, where $N^{\prime}=1 \cdot\left\{\left(\bar{x}, x_{j}, g(t+1), \overline{0}\right)\right\}$, and hence $N^{\prime}$ is $g,(t+2)$-controlled. Therefore by inductive hypothesis we have

$$
\begin{aligned}
P_{g, n}\left(\bar{x}, x_{j}+1, \overline{0}, t\right) & \leq P_{g, n}\left(\bar{x}, x_{j}, g(t+1), \overline{0}, t+2\right) \\
& \leq U_{g, n}\left(\bar{x}, x_{j}, g(t+1), \overline{0}, t+2\right)=U_{g, n}\left(\bar{x}, x_{j}+1, \overline{0}, t\right),
\end{aligned}
$$

and this concludes the proof. 
Theorem 14. If $g$ is primitive recursive and $g(t) \geq t+1$ then $L_{g, n}^{\mathrm{ms}}$ has an upper bound in $\mathfrak{F}_{\omega^{n}}$. Also, this bound is tight.

Proof. The fact that the bound is tight follows from Thm. 12. Without loss of generality suppose, $t>2$ and let $2 \leq e<\omega$ such that $g(t+1) \leq F_{e}(t)$. By $\left(\forall^{\infty} x\right) \varphi(x)$ we mean that $\varphi$ holds for almost every $x$, i.e $(\exists k)(\forall x>k) \varphi(x)$.

Fact 4 If $x \neq 0$ then $\left(\forall^{\infty} t\right)(\forall x) U_{g, n}(\overline{0}, x, t) \leq F_{3(x-1)+e}(t)$.

Proof. By induction in $x \neq 0$. For $x=1$, observe that $U_{g, n}(\overline{0}, 1, t)=g(t+$ $1) \leq F_{e}(t)$. For the inductive step, $o_{\overline{0}, x}=t+U_{g, n}(\overline{0}, x, t) \leq t+F_{3(x-1)+e}(t) \leq$ $F_{3(x-1)+e+1}(t)$. Now

$$
\begin{array}{rlr}
U_{g, n}(\overline{0}, x+1, t) & =g(t+1) \cdot U_{g, n}\left(\overline{0}, x, o_{\overline{0}, x}^{g(t+1)-1}(t+2)\right) \\
& \left.\leq g(t+1) \cdot F_{3(x-1)+e}\left(o_{\overline{0}, x}^{g(t+1)-1}(t+2)\right) \quad \text { (ind. hyp. }\right) \\
& \leq F_{e}(t) \cdot F_{p(x)}\left(F_{p(x)+1}^{g(t+1)-1}(t+2)\right) \quad(p(x) \stackrel{\text { def }}{=} 3(x-1)+e) \\
& \leq F_{e}(t) \cdot F_{p(x)}\left(F_{p(x)+1}^{g(t+1)+1}(g(t+1))\right. \\
& =F_{e}(t) \cdot F_{p(x)}\left(F_{p(x)+2}(g(t+1))\right. \\
& \leq F_{p(x)+2}\left(F_{p(x)+2}\left(F_{p(x)+2}\left(F_{p(x)+2}(t)\right)\right)\right. \\
& =F_{p(x)+2}^{4}(t) \leq F_{p(x)+3}(t)=F_{3 x+e} .
\end{array}
$$

This concludes the proof of the Fact

Fact 5 If $x_{0}>0$ then $\left(\forall^{\infty} t\right)\left(\forall x_{n-1}, \ldots, x_{0}\right)\left[U_{g, n}\left(x_{n-1}, \ldots, x_{0}, t\right) \leq F_{\gamma}(t) \Rightarrow\right.$ $\left.U_{g, n}\left(x_{n-1}, \ldots, x_{0}+1, t\right) \leq F_{\gamma+3}(t)\right]$.

Proof. Same idea as in Fact 4 .

Fact 6 If $x_{i}>0$ for some $i \geq 1$ then $\left(\forall^{\infty} t\right)\left(\forall \bar{x}=x_{n-1}, \ldots, x_{1}\right) U_{g, n}(\bar{x}, 0, t) \leq$ $F_{\alpha}(t)$, where $\alpha=x_{n-1} \cdot \omega^{n-1}+x_{n-2} \cdot \omega^{n-2}+\cdots+x_{2} \cdot \omega^{2}+x_{1} \cdot \omega+1$.

Proof. By induction in $\bar{x} \neq \overline{0} . U_{g, n}(\overline{0}, 1,0, t)=U_{g, n}(\overline{0}, g(t+1), t+2) \leq F_{d(t)}(t+$ $2) \leq F_{d(t)+1}(d(t))=F_{\omega}(d(t)) \leq F_{\omega+1}(t)$ where $d(t) \stackrel{\text { def }}{=} 3(g(t+1)-1)+e$, the first inequality follows from Fact 4 and the last one is true for all $t \geq k_{1}$.

Next, $U_{g, n}\left(\overline{0}, x_{1}+1,0, t\right)=U_{g, n}\left(\overline{0}, x_{1}, g(t+1), t+2\right) \leq F_{x_{1} \cdot \omega+1+r(t)}(t+2) \leq$ $F_{x_{1} \cdot \omega+1+r(t)}(r(t))=F_{\left(x_{1}+1\right) \cdot \omega}(r(t)) \leq F_{\left(x_{1}+1\right) \cdot \omega+1}(t)$ where $r(t) \stackrel{\text { def }}{=} 3 g(t+1)$, the first inequality follows from ind. hyp. and Fact 5 and the last one is true for all $t \geq k_{2} \geq k_{1}$ (independently of $x_{1}$ ).

Finally, let $\bar{x}=x_{n-1}, \ldots, x_{j-1}$ and let $\beta=x_{n-1} \cdot \omega^{n-1}+\cdots+x^{j-1} \cdot \omega^{j-1}$.

$$
\begin{aligned}
U_{g, n}\left(\bar{x}, x_{j}+1, \overline{0}, t\right) & =U_{g, n}\left(\bar{x}, x_{j}, g(t+1), \overline{0}, t+2\right) \\
& \leq F_{\beta+x_{j} \cdot \omega^{j}+g(t+1) \cdot \omega^{j-1}+1}(t+2) \\
& \leq F_{\beta+x_{j} \cdot \omega^{j}+(g(t+1)+1) \cdot \omega^{j-1}}(t+2) \\
& \leq F_{\beta+x_{j} \cdot \omega^{j}+(g(t+1)+1) \cdot \omega^{j-1}}(g(t+1)) \\
& \leq F_{\beta+\left(x_{j}+1\right) \cdot \omega^{j}}(g(t+1)) \leq F_{\beta+\left(x_{j}+1\right) \cdot \omega^{j}+1}(t),
\end{aligned}
$$

where the last inequality is true for all $t \geq k_{3} \geq k_{2}$ (independently of $\bar{x}, x_{j}$ ). 
Now, let $t$ be sufficiently large. If $n=1$ then $L_{g, n}^{\mathrm{ms}}(t) \leq P_{g, n}(g(t), t+$ $1) \leq U_{g, n}(g(t), t+1) \leq F_{3(g(t)-1)+e}(t+1) \leq F_{3(g(t)-1)+e+1}(3(g(t)-1)+e)=$ $\left.F_{\omega}(3(g(t)-1)+e)\right) \in \mathfrak{F}_{\omega}$, where the second inequality follows from Lem. 13 and the third one from Fact 4 . If $n>1$ we have:

$$
\begin{aligned}
L_{g, n}^{\mathrm{ms}}(t) & \leq P_{g, n}(g(t), \overline{0}, t+1) \\
& \leq U_{g, n}(g(t), \overline{0}, t+1) \\
& \leq F_{g(t) \cdot \omega^{n-1}+1}(t+1) \\
& \leq F_{(g(t)+1) \cdot \omega^{n-1}}(g(t))=F_{\omega^{n}}(g(t)) \in \mathfrak{F}_{\omega^{n}} .
\end{aligned}
$$

The second inequality follows from Lem. 13 and the third one from Fact 6 .

\section{The majoring ordering}

Recall from 92 that the underlying order of $\leq_{\text {maj }}$ is $\leq_{\text {pr }}$ and the underlying order of $\leq_{\text {ms }}$ is $\leq_{\text {lex }}$. We linearize the wqo $\left(\mathcal{P}_{<\infty}\left(\mathbb{N}^{n}\right), \leq_{\text {maj }}\right)$ into the well-order $\left(\mathcal{M}_{<\infty}\left(\mathbb{N}^{n}\right), \leq_{\text {ms }}\right)$ and derive an upper bound for $L_{n, g}^{\text {maj }}(t)$, the length of the longest $g, t$-controlled bad sequence of finite sets of $n$-tuples with respect to the majoring ordering $\leq_{\text {maj }}$. To do this, we use the results of $\$ 5$

Our linearization will be done in two steps. Given a $\leq_{\text {maj-bad sequence } \mathbf{X}=}$ $X_{0}, X_{1}, \ldots X_{k}$ of finite and nonempty sets of $n$-tuples we define an intermediate sequence $T_{0}, T_{1}, \ldots, T_{k}$ of trees whose nodes are decorated with $n$-tuples. From these trees we define a sequence of finite and nonempty multisets of $n$-tuples

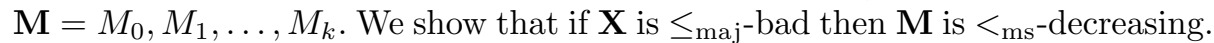
Furthermore, given a control for $\mathbf{X}$, we find a control for $\mathbf{M}$. Using the results of $\$ 5$ we give an answer to the question of the maximum possible length of a

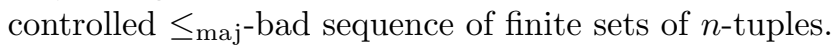

Let $X \subseteq \mathbb{N}^{n}$. We say $X$ avoids $x$ if for all $y \in X$ we have $x \not \mathbb{p}_{\mathrm{pr}} y$. Since $\mathbf{X}=X_{0}, X_{1}, \ldots, X_{k}$ is bad, then for any $i<j, X_{j}$ avoids some tuple of $X_{i}$. In particular for all $j \in\{1, \ldots, k\}, X_{j}$ avoids some tuple of $X_{0}$. If $a$ is the $\leq_{\mathrm{pr}^{-}}$ supremum of $X_{0}$ then $\tilde{\mathbf{X}}=\{a\}, X_{1}, \ldots X_{k}$ is also a bad sequence. Furthermore, if $\mathbf{X}$ was $g, t$-controlled then $\tilde{\mathbf{X}}$ also is, and in this case $a \leq_{\mathrm{pr}}\langle g(t)-1, \ldots, g(t)-1\rangle$. Even more, if $\mathbf{X}$ is the longest such sequence then $a=\langle g(t)-1, \ldots, g(t)-1\rangle$. Therefore, without loss of generality we may assume that all $\leq_{\text {maj-bad }}$ sequences of sets analyzed here have a singleton as the first element.

Construction of the trees $T_{i}$. Without loss of generality suppose $X_{0}=\left\{a_{0}\right\}$. Define the following sequence of finite trees of $n$-tuples. By path we always refer to a path from the root to a leaf. See Fig. 1 for an example of this construction.

$-T_{0}$ is $a_{0}$, the root.

- $T_{i+1}$ is formed by extending $T_{i}$ as follows. For any path $a_{0}, \ldots, a_{m}$ in $T_{i}$ do the following: if for all $j=0, \ldots, m, X_{i+1}$ avoids $a_{j}$ then add all the elements of $X_{i+1}$ as new children of $a_{m}$. 
$\langle 3,3\rangle$<smiles>[13CH3][13CH2][13CH3]</smiles>

$T_{0}$

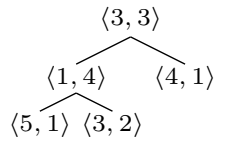

$T_{2}$

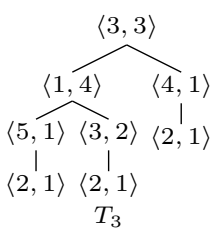

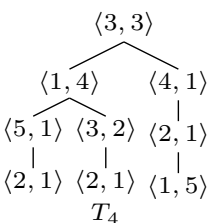

Fig. 1. Construction of the trees for the bad sequence $X_{0}, X_{1}, X_{2}, X_{3}, X_{4}$, where $X_{0}=$ $\{\langle 3,3\rangle\} ; X_{1}=\{\langle 1,4\rangle,\langle 4,1\rangle\} ; X_{2}=\{\langle 5,1\rangle,\langle 3,2\rangle\} ; X_{3}=\{\langle 2,1\rangle\} ; X_{4}=\{\langle 1,5\rangle\}$

Proposition 15. At least one path of $T_{i}$ is strictly extended in $T_{i+1}$.

Proof. Recall that $X_{j} \neq \emptyset$ for all $j$. It is clear that if all internal nodes of $T_{i}$ have a child which is avoided by $X_{i+1}$ then there is a path $a_{0}, \ldots, a_{m}$ in $T_{i}$ such that $X_{i+1}$ avoids $a_{j}$ for all $j$.

If $T_{i+1}=T_{i}$ then, by construction, there is no path $a_{0}, \ldots, a_{m}$ with all of its elements avoided by $X_{i+1}$. Then there is an internal node of $T_{i}$, say $a$, with none of its children avoided by $X_{i+1}$. But this contradicts the badness of $\mathbf{X}$ since by construction the set of children of $a$ is $X_{j}$ for some $j \leq i$.

As the example in Fig. 1 shows, the height of $T_{i+1}$ is not necessarily greater than the height of $T_{i}$. The following follows by construction:

Proposition 16. Any path in $T_{i}$ is a bad sequence of $n$-tuples with respect to the product ordering. Furthermore if $\mathbf{X}$ is $g$, $t$-controlled then any such path is $g,(t+i)$-controlled.

Construction of the multisets $M_{i}$. Let $M_{i} \in \mathcal{M}_{<\infty}\left(\mathbb{N}^{n}\right)$ be defined as: $M_{i}(y) \stackrel{\text { def }}{=} d$ iff there are exactly $d$ paths in $T_{i}$, say $p_{1}, \ldots, p_{d}$, such that $h_{n}\left(p_{j}\right)=y$ for all $j$. In other words, $M_{i}$ is the multiset where we put $h_{n}(p)$ for every path in $T_{i}$.

If the path $\bar{a}=a_{1}, \ldots, a_{m}$ in $T_{i}$ is extended to $\bar{a}, x$ in $T_{i+1}$ then by Thm. 4 , $h_{n}(\bar{a}, x)<_{\operatorname{lex}} h_{n}(\bar{a})$. Then $M_{i+1}<_{\mathrm{ms}} M_{i}$. The need for working with multisets and not simply with sets resides in the fact that $h$ is not injective.

Proposition 17. If $\mathbf{X}=X_{0}, \ldots, X_{k}$ is $g$, t-controlled then $\left|M_{k}\right|<\tilde{g}(t+k)$, for $\tilde{g}(x)=n ! g(n x)^{n(x+1)}+1$.

Proof. Observe that the maximum multiplicity of an element in $M_{k}$ is bounded by $\prod_{j=1}^{k} g(t+j)^{n} \leq g(t+k)^{n k}<\tilde{g}(t+k)$. By Prop. 16 each of such path is $g,(t+k)$-controlled and by the second part of Thm. 4 we have that if $x \in M_{k}$ then $|x|_{\infty}<n ! g(n(k+t))^{n}<\tilde{g}(t+k)$.

Altogether we have shown:

Theorem 18. There is a function $f_{n}:\left(\mathcal{P}_{<\infty}\left(\mathbb{N}^{n}\right)\right)^{+} \rightarrow \mathcal{M}_{<\infty}\left(\mathbb{N}^{n}\right)$ such that if $\mathbf{X} \wedge X$ is a bad sequence in $\left(\mathcal{P}_{<\infty}\left(\mathbb{N}^{n}\right), \leq_{\text {maj }}\right), \mathbf{X}$ is nonempty and $X$ is a nonempty set, then $f_{n}\left(\mathbf{X}^{\wedge} X\right)<_{\mathrm{ms}} f_{n}(\mathbf{X})$. Furthermore if $\mathbf{X}$ is g,t-controlled then $\left|f_{n}(\mathbf{X})\right|<\tilde{g}(|\mathbf{X}|-1+t)$, for $\tilde{g}$ as in Prop. 17 . 
Proof. Take $f_{n}(\mathbf{X})=M_{|\mathbf{X}|-1}$ as in the above construction.

Let $L_{n, g}^{\mathrm{maj}}(t)$ denote the length of the longest $g, t$-controlled bad sequence in $\left(\mathbb{N}^{n}, \leq_{\text {maj }}\right)$, and let $L_{n, g}^{\mathrm{ms}}(t)$ denote the length of the longest $g, t$-controlled decreasing sequence in $\left(\mathbb{N}^{n},<_{\mathrm{ms}}\right)$.

Corollary 19. For any primitive recursive $g$ there is a primitive recursive $\tilde{g}$ such that $L_{n, g}^{\mathrm{maj}} \leq L_{n, \tilde{g}}^{\mathrm{ms}}$. Hence there is an upper bound of $L_{n, g}^{\mathrm{maj}}$ in $\mathfrak{F}_{\omega^{n}}$.

Proof. It follows from Thm. 18 and Thm. 14

\section{Applications}

In Jurdziínki and Lazić [9] it is shown that for the class of incrementing tree counter automata (ITCA) as well as the class of alternating top-down tree one register automata (ATRA), the emptiness problem - i.e. whether the language accepted by an automaton of such classes is empty - is decidable over finite data trees. Figueira [4] later showed that for some extensions of ATRA decidability still holds. All these proofs go along the lines of interpreting the automata execution as a downward well-structured transition system, then showing that it is reflexive-downward-compatible with respect to a wqo between sets of configurations, and finally applying Finkel and Schnoebelen results [6] (mainly Prop. 5.4). That wqo is precisely the majoring order.

From [9], we know that the computational complexity of such decision procedures is lower-bounded by a non-primitive recursive function. For the upperbound for ITCA's, an algorithm can be given in a manner analogous to [5, §VII.B.] for finding the levels (a finite set of configurations) reachable from the initial level - the emptiness problem is then reduced to testing whether the empty level is amongst them. The complexity of such an algorithm is mainly determined by the length of a bad sequence of levels $\mathbf{V}=V_{0}, V_{1}, \ldots, V_{m}$. In more detail, suppose an ITCA $\mathcal{C}$ has $k$ counters and a finite set of states $Q$. Then a level of $\mathcal{C}$ is a finite set of tuples of the form $\langle q, v\rangle$, where $q \in Q$ and $v=\left\langle a_{1}, \ldots, a_{k}\right\rangle \in \mathbb{N}^{k}$ is the current values of the $k$ counters. The levels are ordered by the the majoring ordering with the following underlying order

$$
\langle p, u\rangle \leq\langle q, v\rangle \stackrel{\text { def }}{\Leftrightarrow} p=q \wedge u \leq_{\mathrm{pr}} v,
$$

which is a wqo. The complexity of the emptiness problem can be bounded by the length of the longest bad sequence in $\left(\mathcal{P}_{<\infty}\left(Q \times \mathbb{N}^{k}\right), \leq_{\text {maj }}^{(\leq)}\right)$. As one can see, the application of Cor. 19 is not entirely straightforward because it applies to the majoring ordering of finite sets of tuples of $\mathbb{N}$ with the underlying $\leq_{\text {pr }}$ and not to levels with the underlying $\leq$. We reduce bad sequences of levels to bad sequences of finite sets of tuples as follows. Suppose $Q=\left\{q_{0}, \ldots, q_{s-1}\right\}$ and let $q_{i}^{\prime} \stackrel{\text { def }}{=}(i, s-i) \in \mathbb{N}^{2}$. Clearly if $p^{\prime} \leq_{\mathrm{pr}} q^{\prime}$ then $p^{\prime}=q^{\prime}$ and so $p=q$. Let $V \in \mathcal{P}_{<\infty}\left(Q \times \mathbb{N}^{k}\right)$ be a level. Define $V^{\prime} \stackrel{\text { def }}{=}\left\{\left\langle p^{\prime}, u\right\rangle \in \mathbb{N}^{k+2} \mid\langle p, u\rangle \in V\right\}$. The reader can verify that if $V$ and $W$ are levels then $V^{\prime} \leq_{\text {maj }}^{\left(\leq_{\mathrm{pr}}\right)} W^{\prime}$ implies 
$V \leq_{\text {maj }}^{(\leq)} W$. Hence $\mathbf{V}=V_{0}, V_{1}, \ldots, V_{m}$, a bad sequence of levels of an ITCA with $k$ counters, can be seen as a bad sequence of the same length $\mathbf{V}^{\prime}=V_{0}^{\prime}, V_{1}^{\prime}, \ldots, V_{m}^{\prime}$ in $\mathcal{P}_{<\infty}\left(\mathbb{N}^{k+2}\right)$ with the majoring ordering studied in 86 . Regarding how $\mathbf{V}^{\prime}$ is controlled, the analysis is almost the same as in [5, §VII.B.]. Let $V_{0}^{\prime}=\{\langle 0,|Q|-$ $1, \overline{0}\rangle\}$ and $V_{i}^{\prime}=\left\{c_{1}, \ldots, c_{p_{i}}\right\}$. From Def. 6 we have that $\left|V_{i}^{\prime}\right|=\max _{j}\left\{\left|c_{j}\right|_{\infty}\right\}$. The change from $V_{i}^{\prime}$ to $V_{i+1}^{\prime}$ may involve a change of state or increment of $c_{j}$ 's counters' values by one. The 'state part' of $c_{j}$ is controlled by the constant $|Q|$ and the 'counters part' is controlled by the successor function. Hence, the bad sequence of sets is $g, 0$-controlled by $g(t)=t+1+|Q|$. Now we can finally apply Cor. 19 to conclude that the complexity of the emptiness problem for an ITCA with $k$ counters is upper bounded by a function in $\mathfrak{F}_{\omega^{k+2}}$.

This immediately gives us an upper bound for the emptiness problem for ATRA. From [9, Thm. 3.1] we have that emptiness for ATRA follows from a PSPACE-reduction to emptiness for ITCA. If the ATRA $\mathcal{A}$ has $s$ states then the ITCA $\mathcal{C}$ constructed in the reduction has $k(s) \stackrel{\text { def }}{=} 2^{s}-1+2^{4 s}$ counters 1 Hence the complexity of the emptiness problem for an ATRA with s states is upper bounded by a function in $\mathfrak{F}_{\omega^{k(s)+2}}$.

The above complexities are obtained by the straightforward codification of levels $V$ into $V^{\prime}$. This increases the dimension of tuples from $n$ to $n+2$, and this might be too wasteful. It seems plausible to work directly with levels (i.e. sets of $Q \times \mathbb{N}^{n}$ ) and obtain better upper bounds.

\section{Conclusions}

Upper bounds for controlled descending sequences in a well-order are easier to obtain than for controlled bad sequences in a wqo's. We studied upper bounds for the length of controlled bad sequences of two wqo's by linearizing them into well-orders. Such bounds were placed in the Fast Growing Hierarchy.

For the product ordering of tuples, we gave a straightforward elementary proof for an upper bound of controlled bad sequences, and we arrived to the same general result as [5] but avoiding the "sum of powers of $\mathbb{N}$ " approach. This last approach - being noticeably more understandable than previous proofs, and also leading to a more general result - still needs some rather technical lemmas. Our proof simply relies on a linearization of controlled bad sequences of tuples in the product ordering into controlled descending sequences of tuples in the lexicographic ordering, for which upper bounds can be easily obtained.

For the majoring ordering of sets of tuples, we gave an upper bound of controlled bad sequences over such wqo by linearizing to controlled and descending sequences of multisets with the natural multiset ordering. For the latter we also gave a tight upper bound, which is of interest by itself. As applications we showed complexity upper bounds for the emptiness problem for two types counter automata: ITCA and ATRA.

\footnotetext{
${ }^{1}$ In 9 there is typo in the number of counters in the auxiliary array $c^{\prime}$. Where it says $2^{|Q|^{4}}$, it should read $2^{4|Q|}$.
} 
The fact that $\left(\mathcal{P}_{<\infty}\left(\mathbb{N}^{n}\right), \leq_{\text {maj }}\right)$ is a wqo follows from reducing finite sets of tuples to finite strings over $\mathbb{N}^{n}$ and then applying Higman's Lemma. Schmitz and Schnoebelen [13] developed an algebraic framework for handling normed wqo's where upper bounds for controlled bad sequences when using Higman's Lemma on finite alphabets are derived. Hence, another approach to obtain upper bounds for the majoring ordering would be to try to extend this framework to deal with strings over infinite alphabets.

As future research we will study lower bounds for the majoring ordering, and upper bounds for the bad sequences over the dual of the majoring ordering, the minoring ordering:

$$
A \leq \stackrel{(\leq)}{\min } B \stackrel{\text { def }}{\Leftrightarrow}(\forall y \in B)(\exists x \in A) x \leq y .
$$

This is not in general a wqo: one needs the underlying $\leq$ to be an $\omega^{2}$-wqo 8 , Thm. 1]. It would also be interesting to investigate how far one can generalize this idea of linearization. Are there general ways in which one can relate the length of a bad sequence over a wqo into the length of a linearization of it?

\section{References}

1. S. A. Abriola. Sobre la longitud de las secuencias malas controladas en cuasiórdenes buenos. MSc thesis, Universidad de Buenos Aires, Argentina, 2011.

2. P. Clote. On the finite containment problem for Petri nets. Theoretical Computer Science, 43:99-105, 1986

3. N. Dershowitz and Z. Manna. Proving termination with multiset orderings. Communications of the ACM, 22(8):465-476, August 1979.

4. D. Figueira. Reasoning on Words and Trees with Data. PhD thesis, Laboratoire Spécification et Vérification, ENS Cachan, France, December 2010.

5. D. Figueira, S. Figueira, S. Schmitz, and Ph. Schnoebelen. Ackermannian and primitive-recursive bounds with Dickson's lemma. In LICS, pages 269-278, 2011.

6. A. Finkel and $\mathrm{Ph}$. Schnoebelen. Well-structured transition systems everywhere! Theoretical Computer Science, 256(1-2):63-92, 2001.

7. W. Harwood, F. Moller, and A. Setzer. Weak bisimulation approximants. In Zoltán Ésik, editor, Computer Science Logic. 20th International Workshop, CSL 2006, 15th Annual Conference of the EACSL, volume 4207 of Lecture Notes in Computer Science, pages 365-379, 2006.

8. P. Jancar. A note on well quasi-orderings for powersets. Information processing letters, 72(5-6):155-160, 1999.

9. M. Jurdziński and R. Lazić. Alternating automata on data trees and XPath satisfiability. ACM Transactions on Computational Logic (TOCL), 12(3):19, 2011.

10. M.H. Löb and S.S. Wainer. Hierarchies of number theoretic functions, I. Archive for Mathematical Logic, 13:39-51, 1970.

11. K. McAloon. Petri nets and large finite sets. Theoretical Computer Science, 32(12):173-183, 1984.

12. R. Péter. Recursive functions. Academic Press, 1967.

13. S. Schmitz and Ph. Schnoebelen. Multiply-recursive upper bounds with Higman's lemma. In Proceedings of the 38th international conference on Automata, languages and programming - Volume Part II, ICALP'11, pages 441-452. Springer-Verlag, 2011. 